\title{
Conceptual modelling for water quality management
}

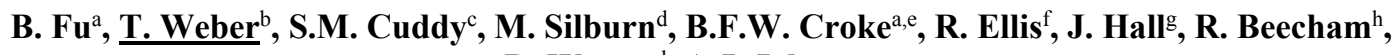 \\ D. Waters ${ }^{\text {d }}$, A.J. Jakeman ${ }^{\text {a }}$ \\ ${ }^{a}$ Fenner School of Environment and Society, Australian National University, Canberra \\ ${ }^{b}$ Alluvium Consulting, Brisbane \\ ${ }^{c}$ CSIRO Land and Water, Canberra \\ ${ }^{d}$ Queensland Department of Natural Resources and Mines, Toowoomba \\ e Mathematical Sciences Institute, Australian National University, Canberra \\ ${ }^{f}$ Queensland Department of Science, Information Technology and Innovation, Brisbane \\ ${ }^{g}$ Western Australia Department of Water, Perth \\ ${ }^{h}$ New South Wales Department of Primary Industries, Sydney \\ Email:tony.weber@alluvium.com.au
}

\begin{abstract}
Water quality models are replete with implicit assumptions. Some of the assumptions may be legacy in nature, having originated from early development of a model and subsequently taken for granted within the scientific community. For instance, Easton et al. (2008) reported that the SWAT model implicitly assumes an infiltration-excess response to rainfall when predicting storm runoff, which is not applicable to humid, well vegetated regions and where saturation-excess is the dominant process. The re-conceptualisation of the SWAT model then led to the development of a new modelling approach SWAT-VSA.
\end{abstract}

Development of an appropriate suite of conceptual models should be one of the first steps in water quality modelling - appropriate in the sense that they capture key operating processes (runoff generation, transport and delivery) and landscape connectivities within catchments. The history of water quality modelling is one of evolution with most of the popular models built on, or adapted from, existing models or experimental studies. As a consequence, the conceptualisations underpinning these models are rarely scrutinised sufficiently.

In this paper, we use the eWater Source modelling framework as a case study for critically analysing conceptualisations of constituent generation and transport fluxes. We have selected this product as its water quality modelling component is currently being enhanced and our analysis can usefully inform its future. Our observations, which are pertinent to many water quality models, include:

- The current filtration and transport processes are basic representations. Depending on the water quality parameters being modelled, these may not be sufficient to capture the range of processes needing to be quantified in catchment modelling projects.

- The base spatial unit for generation is an unlinked functional unit (FU), which is a property-based semidistributed approach to spatial discretisation. This limits the ability to capture important factors such as the distance of FU to streams, drainage/channelisation on FUs and relationships between FUs in the same subcatchment.

- The temporal scale for constituent generation is generally daily. Moving to sub-daily scales may be needed to better capture the influence of rainfall intensity, especially in urbanised catchments.

- Some aspects of management impacts are not adequately considered, such as lag-time between catchment management actions and system response.

It could be argued that many of these conceptualisations are simplifications designed to balance complexity with usability and computational efficiency. Nevertheless, they need to be challenged and alternatives explored if we are to advance the science and practical effectiveness of water quality modelling. In this paper we report our initial investigations and propose some potential improvements in conceptualisation to allow for better representation of the system for water quality management.

Keywords: Conceptual modelling, water quality, eWater Source, water quality modelling, generation, delivery and transport processes 


\section{INTRODUCTION}

Conceptual modelling is the process of abstracting a model from a real or proposed system (Robinson, 2008). A conceptual model is the mathematical and logical representation of the problem entity developed for a particular study (Sargent, 2013), often comprising a system diagram that links variables from drivers to internal states to outputs. They may also indicate scales of representation and types of processes deemed relevant. Conceptual models are foundational for the subsequent simulation or computational model which implements the conceptual model on a computer system such that model behaviour, and scenario, analyses can be conducted. Developing appropriate conceptual models is thus a vital step in modelling to ensure the model representation of the system and problem entity is reasonable for the model purpose in question. When evaluating a conceptual model, Robinson (2008) suggests four criteria: validity, credibility, utility and feasibility. Both validity and credibility require the conceptual model to be a sufficiently accurate representation of the system and problem domain for the purpose - the former is from a modeller's perspective, and the latter from a client's perspective. Utility emphasises the conceptual model can be developed into a computer model that is useful as an aid to decision-making (e.g. ease-of-use, parameterisability, flexibility, run time). Feasibility requires that the conceptual model can be developed into a computer model with the time, resources and data available.

In Australia, the eWater Source (Catchments) software has been developed to serve as Australia's national hydrological modelling platform, and has been designed as an integrated catchment, river system planning and operations tool. Since its release, the bulk of its development has been to meet requirements of the member States and Territories, largely focussed on quantitative water sharing and management arrangements. While it is widely agreed that Source brought a fresh approach to catchment modelling through its modular architecture and integration of catchment with river system modelling, its in-built constituent models (including constituent generation, filtration and in-stream transport) are basic (though they can be replaced by using software plugins). These models reflect conceptualisations used in many water quality models of the past few decades. It also has a rudimentary interface for describing catchment management practices which affect water quality. When the various simplifications are put together, the ability to model catchments and its management practices at a level to be able to sufficiently quantify the quality of water moving through that landscape and to support the evaluation of management practices and catchment investment decisions is compromised.

As a step towards improving the capability of constituent models for catchment management in Australia, we re-examine the conceptualisation of the constituent models, using eWater Source as our case study. We firstly review some of the underlying assumptions in its constituent modelling conceptualisation. From that, we identified seven issues to further examine, as being most relevant in the context of using the models to support catchment management investment decisions. We also propose potential improvements to these conceptualisations, with considerations of the model validity, credibility, utility and feasibility.

\section{CONCEPTUALISATION OF WATER QUALITY MODELS}

Water quality models have been developed over many decades. They have been applied to many different types of water systems including streams and rivers (regulated and unregulated), urban systems, lakes, reservoirs, estuaries, coastal waters, and oceans. Here we focus on catchment-scale models of freshwater, non-urban systems. Examples of commonly used catchment-scale water quality models include Soil and Water Assessment Tool (SWAT) (Arnold et al., 2012), Hydrologic Simulation Program - Fortran (HSPF) (Bicknell et al., 2001), Water Erosion Prediction Project (WEPP) (Flanagan et al., 2007), and the Sediment river Network model (SedNet) (Wilkinson et al., 2009). Some components of these models have been implemented or plugins written for the Source platform.

In general, there are three main processes to be considered when modelling the fate of constituents in a catchment: (i) generation, (ii) filtration/delivery from sources to channels and (iii) in-stream transport (which may include deposition, remobilisation and transformation). Most of the commonly used models attempt to represent one or more of these main processes, with different conceptualisations. For example, conceptualisations of the constituent generation process range from using (i) characteristic export rates (e.g. mass/ha/yr from a landuse), (ii) event mean concentration (EMC) and dry weather concentration (DWC) to capture event-based processes, to (iii) representing more detailed physical processes of constituent generation. In terms of the filtration/delivery process, conceptualisations range from using (i) a constant (e.g. delivery ratio), (ii) a function of attribute(s) such as vegetation or terrain, to (iii) more detailed pathways of movement and transformation. In-stream transport process can be represented through empirical routing functions, or more detailed algorithms that account for complex interactions of constituents. Many of the commonly used models have been reviewed in various literature (Merritt et al., 2003; Migliaccio and Srivastava, 2007; SKM, 2011). Notably, Merritt et al. (2003) reviewed 17 erosion and sediment transport models in relation to their 
complexity, inputs, outputs, processes and scales. One observation is the general limitations in representing delivery and transport processes in many models. This is echoed in a more recent review (SKM, 2011). Migliaccio and Srivastava (2007) compared the representation of hydrological processes in six commonly used catchment-scale water quality models. They found that representation of groundwater components is one of the most variable hydrologic components in these models. They also reported that the primary differences in the infiltration and surface runoff algorithms among these models are their empirical (e.g., curve number and Green-Ampt) or physical (e.g., Philip's) basis and their simulation time step. These differences show different conceptualisations of runoff pathways in the landscape. While simpler models have their advantages, they tend to have limitations when implementing changes in management practices; for example, what are the effects of changes in management on EMC and DWC?

Spatial discretisation is a key aspect in the conceptualisation of catchment-scale models. Discretising the modelled area spatially can help identify the sources of constituents that contribute disproportionately high export rate relative to the surrounding area (e.g. 'hot spots', or ecosystem control points (Bernhardt et al., 2017)). Identifying these areas can assist in targeting management investment and monitoring. Typically many catchment-scale models use either a fully distributed (e.g. gridded) approach or use hydrological connectivity to break the catchment into subcatchments. A node-link structure is then created with nodes representing subcatchments and links representing river reaches. Within a subcatchment, Arnold et al. (2010) identified four major types of spatial discretisation:

a) No discretisation or a lumped approach, where the subcatchments are represented by a fixed set of properties (e.g. dominant soil, landuse, slope). In this approach, changes (e.g. management actions) exerted at one part of the subcatchment cannot be accounted for (Figure 1).

b) Semi-distributed approach based on landuse, soil and topographic properties (e.g. slope). Examples are SWAT that uses Hydrologic Response Units (HRUs) (Arnold et al., 2012) and Source that uses Functional Units (FUs) (Wilkinson et al., 2009). Here, a subcatchment is separated into spatially disconnected individual areas of similar soil, landuse and topography. With this approach, interactions between HRUs/FUs are not considered, and thus the impact of management at upslope HRUs/FUs is indifferent to the management occurred at downstream HRUs/FUs.
Conceptualisation

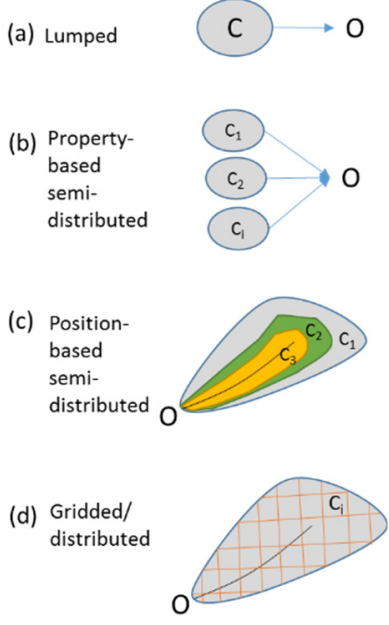

Figure 1. Four main types of spatial discretisation in subcatchments. $\mathrm{C}=$ subcatchment, $\mathrm{O}=$ outlet.

c) Semi-distributed approach based on topographic positions, or catena approach, where a representative hillslope is used to represent the landscape in a subcatchment. For example, a subcatchment may be separated into three spatially connected landscape units: upslope, midslope, and valley bottom areas. This approach assumes uniform properties within a landscape unit, which can be problematic if the landscape unit covers different climate regions, or contains variable slope conditions.

d) Fully distributed approach where a subcatchment is divided into grids or other elements such as triangular elements. This approach requires significant amounts of data and computational requirements and is not considered feasible for large catchments (Pignotti et al., 2017).

Temporal heterogeneity of constituent fluxes is another important aspect in the conceptualisation of constituent models, in part driven by different flow pathways. One typical phenomenon is that constituents can display disproportionally high export rates for a short period of time. McClain et al. (2003) termed this phenomenon as 'hot moments'. This behaviour is typical of constituents which are applied and decay over time, such as fertilisers and pesticides. Similar to 'hot spots', identifying 'hot moments' can help target management interventions. In model conceptualisation, this requires the selection of suitable temporal resolutions and incorporating other temporal dynamics processes in the models that are scientifically valid, useful for management and feasible in terms of resource and data availability. For example, most existing models, especially the catchment-scale models, typically operate at daily scale or coarser. However, it is well recognised 
that in areas with high rainfall intensity, daily flow cannot adequately capture the 'hot moments' of constituent generation and transport (Yang et al., 2016). On the other hand, sub-daily data are generally not available for most areas, especially for large catchments, and thus modelling constituent flux at sub-daily scales is often not feasible. From a catchment management point of view, one typical implication is the inability to capture the impact of timing of interventions, such as the timing of fertilisation in relation to the timing of subsequent rainfall events, but this would obviously be limited by the time step used within the model. In a more recent development of SWAT, sub-daily processes, such as weather and infiltration processes and channel routing, have become more prominent. This functionality is generally applied for modelling urban stormwater management or small agricultural land (e.g. Maharjan et al., 2013).

\section{CONSTITUENT MODELLING CONCEPTUALISATION IN SOURCE}

eWater Source, as a modelling platform, includes several constituent models that users can select for their modelling purposes. We have identified six aspects of constituent modelling conceptualisation, as implemented in Source, that have potential for improvement. Within this paper we briefly cover four of these (1-4). Aspects 5-7 will be considered in another publication.

1. the representation of filtration and transport processes (\% reduction, exponential decay)

2. using a property-based semi-distributed approach to spatial discretisation (the functional unit, FU), which limits the ability to capture important factors such as the distance of the FU to streams, drainage/channelisation on FUs and inter-relationships between FUs

3. using a daily time step for constituent generation, which may not adequately account for rainfall intensity, especially in urbanised catchments

4. some aspects of managing impacts not adequately represented, such as no lag-time between catchment management actions and system response

5. restricting constituent association to FUs or inflow nodes; constituents cannot be associated with other components, such as rainfall

6. restricting the application of the filtration model to the generation of constituents from an FU; it cannot be applied downstream of an FU such as placement of a wetland downstream of a mixed land-use catchment

7. hydrologic flow pathways are not adequately represented, and thus contrasting dynamics of the hydrological connectivity at different source areas is not explicitly accounted for (Hrachowitz et al., 2016).

Note that the models reviewed here are those readily available in the Source core platform (v4.1) (eWater, 2017). We also refer to the recent development in Great Barrier Reef (GBR) Source models such as the Dynamic SedNet plugins (Ellis and Searle, 2014), which offer ideas for ways forward.

\subsection{Filtration and transport processes}

Four filter models have been developed for Source:

- $\quad$ Fixed percentage removal

$\circ$ at each time step, a fixed percentage of the constituent associated with the quick flow of the FU and a fixed percentage of the constituent associated with the baseflow are removed

- $\quad 1$ st Order Kinetic Model k-C*

○ adapted from the universal stormwater treatment model (Wong et al., 2002), this model simulates the decay or reduction in inflow concentration as a function of the 'background concentration' of the treatment facility (e.g. grass strip, wetland), the inflow, and the treatment area

- Load-based sediment or nutrient delivery ratio

○ uses simple rule-based relationships between sediment/nutrient reduction and their loads and rulebased thresholds

- $\quad$ Riparian Particulate model for riparian buffers

$\circ$ accounts for settling of coarse particulates and trapping of fine particulate through infiltration and adhesion at the FU and daily scales. It empirically simulates the loss of particulate constituents using characteristics of buffer, soil vegetation and flow, mostly through multiplication. Up to 28 parameters are required, many of which can have significant spatial variations at FU scale (e.g. stem radius and density, daily vegetation growth rate, depth of unsaturated porous soil layer). 
For transporting constituents, a closed system (no other sources/sinks than what is already considered) is assumed for constituent budgets within a catchment, which may not be valid for many situations, including: 1) when atmospheric dust and chemicals from other catchments and/or in rainfall or groundwater flow become important sources; 2) when a constituent is non-conservative (e.g. loss of $\mathrm{N}$ to the atmosphere, uptake of nutrients by vegetation); or 3) inactivation of pathogens (e.g. bacteria, viruses, protozoa).

Constituents can decay (e.g. exponentially) - however exchanges between constituents are not supported. In part, this is because features which affect these processes, such as surface roughness, may not be well mapped or may be transient. Constituents cannot be related (e.g. forms of nitrogen). Disconnected parts of the landscape are assumed to not contribute runoff to streams. Disconnectedness varies with event size. It is likely that in the large events which export the greatest loads, the vast majority of the catchment is connected to stream.

There are several improvements that may be possible here. Developing plugins for addressing key constituent generation processes may be the most comprehensive solution, but may introduce further model complexity. An approach which exposes constituents to relationships between each other may have the ability to relate the filtration of one constituent to another (e.g. fine and coarse sediment, nutrient transformations). The ability to filter or transform constituents once they are within the hydrologic network, other than simple routing relationships, would allow for more flexibility in management practice simulation. Exposing both the generation and filtration process to allow user-developed functions would significantly improve functionality. Additionally, the use of a different spatial discretisation (e.g. banded approach) may support the simulation of a richer range of filter and transport processes.

\subsection{Functional Unit as base spatial unit}

Functional Units (FUs) represent areas within subcatchments that have similar hydrological behaviours characterised by different properties such as landuses, landscape condition and soil. The convention tends to use landuse, for example forest, grazing, cropping and urban areas. While many models associate a suite of models with a landuse type (e.g. forest), within Source, each FU can have its own suite of rainfall-runoff, constituent generation and filtration models. Each FU independently contributes to constituents at the subcatchment outlet, i.e. they are not linked, and they generally have no spatial references. This semi-distributed conceptualisation of FUs limits the ability to capture important factors such as the distance of a FU to streams or drainage/channelisation on FUs. In addition, without spatial connectivity of the FUs, potential through-flow entering a FU from neighbouring upland FUs cannot be accounted for. From a management perspective, 'knowing' the position of a FU can inform the impacts of location of management actions such as landuse changes. Similarly, knowledge of drainage or channelization structures within a FU (e.g. horticulture drainage system) is important in managing constituent generation and transport, but may not be captured in a subcatchment-scale stream network which is generally much at a coarser scale, being generated from a catchment map or Digital Elevation Model (DEM).

Note that the number of FUs that can be specified in a catchment model is not limited. Thus in theory, FUs can be defined in such detail as to capture the factors relating to spatial positions of the FUs, e.g. differentiating areas (FUs) of cropping near stream from areas of cropping away from stream. However if data to differentiate the constituent properties of such fine-scale FUs are not available, there may be little advantage gained at the cost of potentially long model run times limiting the utility of the model for simulating management scenarios.

One potential improvement could be to develop a hybrid spatial discretisation approach between propertybased and position-based semi-distributed approaches. This would need to balance model utility (i.e. not too fine as to significantly impact model run time) and credibility (i.e. still incorporate important factors to be able to identify 'hotspots' and evaluate management in these areas). An approach proposed by Ning et al. (2015) involves the generation of spatially continuous HRUs (the base unit used in SWAT, similar to FUs in Source) in a subcatchment with explicit hydrological properties (land cover, soil type and slope) and a specific location (barycentre). This approach may be adapted for Source catchment models to account for the position property of the FUs.

Similarly, a specific FU could be defined to capture FUs that have a distinct drainage structure. Another option to capture drainage/channelization in FUs would be to include these features in the stream network. However, this may also result in the definition of a large number of subcatchments and long run times, many of which may not be necessary (e.g. in grazing properties as opposed to horticulture areas).

This is a fruitful area for improvement and there are many studies reported in the literature comparing the efficacy and overheads of different spatial arrangements to give guidance. 


\subsection{Temporal scale of constituent generation}

Temporal scale is probably one of the most vexing aspects of constituent modelling, is a key driver in model conceptualisation, and is most dependent on the constituents of interest and the management questions being asked. For example, if the interest is in long-term loads of a conservative constituent, then a daily time-step (or even monthly) may be sufficient. However, moving to sub-daily scales may be needed to better capture the influence of rainfall intensity, especially in urbanised catchments. As it is becoming common for catchment models to be coupled with receiving water models (which are usually of a fine temporal scale e.g. minutes), the temporal scale of catchment models is being challenged. The Source architecture supports sub-daily time steps. But the prevailing paradigm is daily and most of the routines in Source, including its calibration methods, have been developed and tested using daily data. Rather than focus on this as a limitation, it demonstrates the pervasiveness and influence of the underlying conceptualisation and its inherent biases. As an example, an experiment to calibrate sub-daily models for the Brisbane River catchment was challenging due to long processing time, with the calibration dominated by larger daily events rather than sub-daily short term responses (Weber and Gibbes, 2016). Work is currently underway to improve the temporal scale of constituent generation. For example, the Queensland Water Modelling Network Program is currently investigating current methods of generating rainfall intensity data to supply peak runoff rates in erosion models such as the USLE.

However, we must note that the principle of parsimony should be maintained in the conceptualisation. In this case, from a utility point of view, using sub-daily simulations in water quality for catchments can bring in a huge amount of new overheads (e.g. input data processing, runtimes, data storage and post-processing, calibration data, calibration timeframe). From a feasibility point of view, sub-daily simulations require longterm temporal resolution rainfall records and flow gauge data, which are often not available in most areas. Therefore, the requirement to increase temporal scale of constituent generation should be assessed at case by case basis.

\subsection{Representing management impacts}

There is some capability for modelling management impacts in Source, such as riparian buffer and point source management. However, there is room for improvement. For example, in situations where there has been a build-up of a constituent in a water body (e.g. salt, phosphorus, heavy metals), there will be a lag time in the system response to changes in management practices which address delivery of the constituent to the water body. The lag time will depend on the change in the load delivered to the water body relative to the store of the constituent in the water body and bed sediments. Groundwater systems can have a long lag time due to the amount of a constituent stored in an aquifer and the input and output flux rates. This can include saline aquifers as well as contaminated aquifers (e.g. due to use of firefighting foams that contain perflourooctane sulfonate (PFOS) and perflourooctanoic acid (PFOA)).

There is also an issue in relation to the time for the treatment measure to reach full effectiveness, such as a revegetation project. Across a catchment, levels of adoption and compliance are other factors that affect the modelling of constituent processes, which can be considered in the conceptualisation. Water quality models often need to be able to capture the lag in the impact of management options as well as their cost effectiveness in order to assist in deciding which options are suitable.

Like many other such models, eWater Source defaults to a static scenario approach to exploring the likely impacts of changes in land use and/or management practices. This is an area of active development within Source. For example, a time-series of land use maps can be used to simulate changing land use during the period of the model run.

\section{CONCLUSION}

This paper represents preliminary efforts of the authors to advance the modelling of water quality in Australia, using the eWater Source platform as its case study. Source, like many of its peers, is an expression of the underlying conceptualisation of how catchments work, and in particular how water quality and its constituent processes work. As the science and evidence improves, this conceptualisation, with its inherent assumptions and biases, needs to be challenged and areas for advancement identified.

The advantage of using a product such as Source is that it is amenable to being challenged. Its modular architecture has influenced the design of many of its component models, making it relatively easy to isolate conceptualisation aspects for scrutiny. From this perspective, the independence of the FUs, and the lack of connectivity between some of the component parts, are an advantage as they make it easier to consider and test improvements than if the conceptualisation were internally inter-twined with feedback loops and multiple links. 
Fu et al., Conceptual modelling for water quality management

\section{ACKNOWLEDGMENTS}

This project is funded by the Australian and Queensland Governments through the Reef Plan and the Queensland Water Modelling Network, as well as the Western Australia Government Department of Water and the New South Wales DPIW.

\section{REFERENCES}

Arnold, J., Allen, P., Volk, M., Williams, J. \& Bosch, D. (2010). Assessment of different representations of spatial variability on SWAT model performance. Transactions of the ASABE, 53, 1433-1443.

Arnold, J.G., Moriasi, D.N., Gassman, P.W., Abbaspour, K.C., White, M.J., Srinivasan, R., Santhi, C., Harmel, R., Van Griensven, A. \& Van Liew, M.W. (2012). SWAT: Model use, calibration, and validation. Transactions of the ASABE, 55, 1491-1508.

Bernhardt, E.S., Blaszczak, J.R., Ficken, C.D., Fork, M.L., Kaiser, K.E. \& Seybold, E.C. (2017). Control Points in Ecosystems: Moving Beyond the Hot Spot Hot Moment Concept. Ecosystems, 20, 665-682.

Bicknell, B.R., Imhoff, J.C., Kittle Jr, J.L., Jobes, T.H., Donigian Jr, A.S. \& Johanson, R. (2001). Hydrological simulation program-fortran: HSPF, version 12 user's manual. AQUA TERRA Consultants, Mountain View, California.

Easton, Z.M., Fuka, D.R., Walter, M.T., Cowan, D.M., Schneiderman, E.M. \& Steenhuis, T.S. (2008). Reconceptualizing the soil and water assessment tool (SWAT) model to predict runoff from variable source areas. Journal of hydrology, 348, 279-291.

Ellis, R.J. \& Searle, R.D. (2014). Dynamic SedNet Component Model Reference Guide, Concepts and algorithms used in Source Catchments customisation plugin for Great Barrier Reef catchment modelling. Bundaberg, Queensland: Queensland Department of Science, Information Technology, Innovation and the Arts.

eWater. (2017). Source User Guide 4.1 [Online]. Available: https://wiki.ewater.org.au/display/SD41/Source+User+Guide+4.1 [Accessed 17/8/2017.

Flanagan, D.C., Gilley, J.E. \& Franti, T.G. (2007). Water Erosion Prediction Project (WEPP): Development history, model capabilities, and future enhancements. Transactions of the ASABE, 50, 1603-1612.

Hrachowitz, M., Benettin, P., Van Breukelen, B.M., Fovet, O., Howden, N.J., Ruiz, L., Van Der Velde, Y. \& Wade, A.J. (2016). Transit times - the link between hydrology and water quality at the catchment scale. Wiley Interdisciplinary Reviews: Water, 3, 629-657.

Maharjan, G.R., Park, Y.S., Kim, N.W., Shin, D.S., Choi, J.W., Hyun, G.W., Jeon, J.-H., Ok, Y.S. \& Lim, K.J. (2013). Evaluation of SWAT sub-daily runoff estimation at small agricultural watershed in Korea. Frontiers of Environmental Science \& Engineering, 7, 109-119.

McClain, M.E., Boyer, E.W., Dent, C.L., Gergel, S.E., Grimm, N.B., Groffman, P.M., Hart, S.C., Harvey, J.W., Johnston, C.A., Mayorga, E., McDowell, W.H. \& Pinay, G. (2003). Biogeochemical Hot Spots and Hot Moments at the Interface of Terrestrial and Aquatic Ecosystems. Ecosystems, 6, 301-312.

Merritt, W.S., Letcher, R.A. \& Jakeman, A.J. (2003). A review of erosion and sediment transport models. Environmental Modelling \& Software, 18, 761-799.

Migliaccio, K.W. \& Srivastava, P. (2007). Hydrologic components of watershed-scale models. Transactions of the ASABE, 50, 1695-1703.

Ning, J., Gao, Z. \& Lu, Q. (2015). Runoff simulation using a modified SWAT model with spatially continuous HRUs. Environmental Earth Sciences, 74, 5895-5905.

Pignotti, G., Rathjens, H., Cibin, R., Chaubey, I. \& Crawford, M. (2017). Comparative Analysis of HRU and Grid-Based SWAT Models. Water, 9, 272.

Robinson, S. (2008). Conceptual modelling for simulation Part I: definition and requirements. Journal of the Operational Research Society, 59, 278-290.

Sargent, R.G. (2013). Verification and validation of simulation models. Journal of Simulation, 7, 12-24.

SKM 2011. Development of WQ modelling framework for the MDB Phase 1-scoping study. Final report to the Murray Darling Basin Authority by Sinclair Knight Merz.

Weber, T. \& Gibbes, B. (2006). Pushing the boundaries in Source - subdaily timesteps and improving stream routing. Source 2016, 2016 Canberra. eWater.

Wilkinson, S.N., Prosser, I.P., Rustomji, P. \& Read, A.M. (2009). Modelling and testing spatially distributed sediment budgets to relate erosion processes to sediment yields. Environmental Modelling \& Software, 24, 489-501.

Yang, X., Liu, Q., He, Y., Luo, X. \& Zhang, X. (2016). Comparison of daily and sub-daily SWAT models for daily streamflow simulation in the Upper Huai River Basin of China. Stochastic Environmental Research and Risk Assessment, 30, 959-972. 